\title{
General Impedance Synthesizer Using Minimal Configuration of Switching Converters
}

\author{
Joe C.P. Liu* ${ }^{* \dagger}$ Chi K. Tse ${ }^{\dagger} \quad$ Franki N.K. Poon* ${ }^{*} \quad$ M.H. Pong ${ }^{\ddagger} \quad$ Y.M. Lai ${ }^{\dagger}$
}

\begin{abstract}
A general impedance synthesizer using a minimum number of switching converters is studied in this paper. We begin with showing that any impedance can be synthesized by a circuit consisting of only two simple power converters, one storage element (e.g., capacitor), and one dissipative element (e.g., resistor) or power source. The implementation of such a circuit for synthesizing any desired impedance can be performed by (i) programming the input current given the input voltage such that the desired impedance function is achieved; (ii) controlling the amount of power dissipation (generation) in the dissipative element (source) so as to match the required active power of the impedance to be synthesized. Then, the instantaneous power will automatically be balanced by the storage element. Such impedance synthesizers find a lot of applications in power electronics. For instance, a resistance synthesizer can be used for power factor correction (PFC), a programmable capacitor or inductor synthesizer (comprising of small high-frequency converters) can be used for control applications.
\end{abstract}

\section{INTRODUCTION}

Many problems in electrical engineering are reducible to one of impedance synthesis or imitation [1]. Basically, the impedance observed from the terminals of a given circuit is defined as the ratio of the voltage across the terminals and the current flowing into and out of the terminals, as shown in Fig. 1. In power electronics, for instance, impedance imitation is central to many applications, be it known or clearly recognized by the engineers. Power factor correction (PFC), for example, effectively requires the input impedance to be resistive [2]. Thus, imitating a resistor for the input of a converter is the basic requirement for achieving a high power factor for the converter. Many control problems can also be interpreted as a kind of impedance modification, e.g., shaping the load transient of a converter is essentially a process of modifying the output impedance of the converter [3]. In this paper, we consider the problem of synthesizing impedance using a minimal configuration of switching converters. The problem is relevant to power electronics, and may also be applicable to other branches of electrical engineering. For instance, programmable reactive components using some high-

*PowerELab Limited, c/o University of Hong Kong, Pokfulam, Hong Kong, e-mail: [cpliu,nkpoon] @eee.hku.hk.

$\dagger$ Department of Electronic and Information Engineering, Hong Kong Polytechnic University, Hunghom, Hong Kong, e-mail: [encktse, enymlai] @polyu .edu.hk.

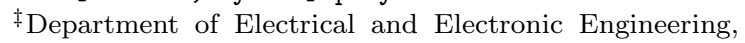
University of Hong Kong, Pokfulam, Hong Kong, e-mail: mhp@eee.hku.hk.

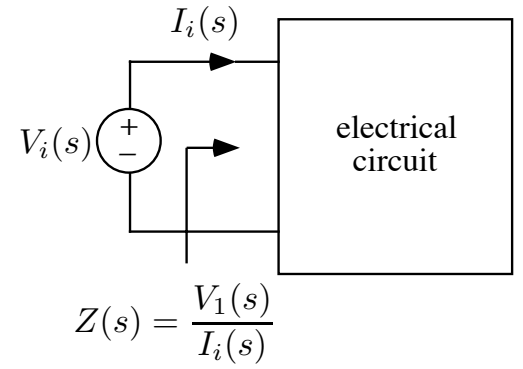

Figure 1: Impedance definition. $Z(s)$ is the impedance observed at the input terminals of the given electrical circuit.

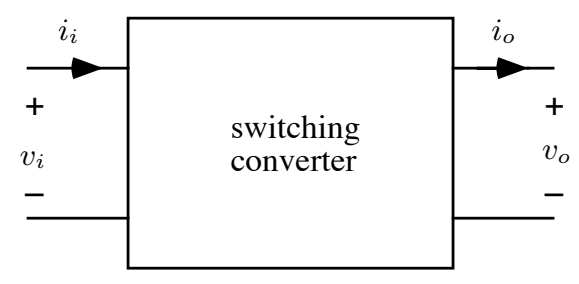

Figure 2: Simple switching converter as two-port.

frequency converters are useful elements for control applications.

We will begin with a review of switching converters in Section 2, with particular emphasis on their fundamental functions in terms of terminal voltages and currents. The main task, to be presented in Section 3, is to find the minimal configuration for synthesizing any impedance. Then, in Section 4, we will present a design of such an impedance synthesizer, and provide some experimental results in Section 5.

\section{REVIEW OF SWITCHING CONVERTERS}

We consider a simple switching converter as a two-port circuit, as shown in Fig. 2. Suppose the input voltage, input current, output voltage and output current are $v_{i}$, $i_{i}, v_{o}$ and $i_{o}$, respectively. In practice, a simple switching converter may contain a storage element and a pair of switches, such as the buck, buck-boost and boost converters. The switches are switched periodically at a high frequency (usually hundreds of $\mathrm{kHz}$ ). The switching frequency can be regarded as being so high that all variables $v_{i}, i_{i}, v_{o}$ and $i_{o}$ are relatively slowly varying. ${ }^{1}$

${ }^{1}$ This assumption holds for all power electronics applications. Ideal switching converters therefore operate at infinite switching frequency. 
Precisely, we write

$$
f_{\max } \ll f_{s}
$$

where $f_{\max }$ is the maximum frequency of $v_{i}, i_{i}, v_{o}$ and $i_{o}$, and $f_{s}$ is the switching frequency of the converter. Specific power processing functions are achieved by adjusting or controlling the relative durations of the "on" and "off" intervals of the switches.

The most fundamental property of an ideal switching converter is the conservation of power. Clearly, the average input power over one repetition period (also called switching period) must equal the average output power over the same period, i.e., $\int_{T} v_{i} i_{i} d t=\int_{T} v_{o} i_{o} d t$. Thus, the ideal converter does not store or dissipate any energy over a repetition period. ${ }^{2}$ Of course, real converters are never $100 \%$ efficient, and here, we ignore this loss to keep our discussion simple. Moreover, in practice, we only consider $v_{i}, i_{i}, v_{o}$ and $i_{o}$ being slowly varying, i.e., varying at a frequency which is much lower than the switching frequency of the converter. Hence, the ideal power conservation equation becomes

$$
v_{i} i_{i}=v_{o} i_{o}
$$

where $v_{i}, i_{i}, v_{o}$ and $i_{o}$ can be regarded as "instantaneous" variables if they satisfy (1).

In addition to the power conservation property, a defining objective of a switching converter is that given one of the terminal variables (normally the input voltage $v_{i}$ ), a switching converter is able to regulate or program one of the remaining three terminal variables by controlling some parameter. For example, for dc power supplies, the input voltage is given, and the switching converter aims to regulate the output voltage by controlling the duty cycle. Moreover, for power factor correction, the input voltage is given (normally a rectified sinewave), and the switching converter aims to program the input current such that it varies at the same frequency and in phase with the input sinewave voltage.

Note that the above definition implicitly assumes that all terminal voltages and currents are slowly varying compared to the switching frequency. Thus, theoretically, if the switching frequency approaches infinity, the switching converter can process voltage or current of arbitrarily high frequencies.

\section{MINIMAL CONFIGURATION OF IMPEDANCE SYNTHESIZER BASED ON SWITCHING CONVERTERS}

In finding the basic configuration of an impedance synthesizer, we first observe that an impedance can be realized by programming the current if the voltage is given, and vice versa. This fits the definition of the switching converter described in Section 2. Thus, a switching converter can be used to program the input current $i_{i}$

\footnotetext{
${ }^{2}$ For any of the simple converters, viz. buck, boost and buck-boost converters, the inductor stores no energy over one switching period.
}

given its input voltage $v_{i}$ in order to create the desired impedance seen from the converter's input. ${ }^{3}$ Here, both $v_{i}$ and $i_{i}$ are slowly varying in the sense of (1). Moreover, the switching converter is subject to the constraint of (2), i.e., power conservation. Clearly, no impedance can be synthesized with one converter terminated with a fixed load impedance because (2) can never be satisfied. ${ }^{4}$ Thus, one switching converter is insufficient. Clearly, we need to balance the power by ensuring that the output of the converter "emits" the right amount of instantaneous power.

If two switching converters are available, one of them can be used to program the input current (given the input voltage) so as to achieve the desired impedance. The other can then be used to match the power conservation requirement by controlling its output to dissipate or generate the correct amount of real power since $v_{i} i_{i}=v_{o} i_{o}$ must be satisfied by both converters. This is possible according to the definition of the switching converter described in Section 2 that, given the load (a resistor or a dc current source), the converter can adjust/control its output voltage $v_{o}$ so as to emit or absorb a desired amount of dc power to or from the output port. Clearly, there must then exist a storage element connecting between the two converters in order to absorb the right amount of instantaneous power to meet the power balance for both converters. Thus, the minimum configuration consists of

- two switching converters,

- one storage element (at frequencies up to $f_{\max }$ ),

- one dissipative element or power source.

Obviously the dissipative element can be realized by a negative power source (e.g., current load). Furthermore, because power flow can be in either directions, the converters must be bi-directional.

\section{IMPLEMENTATION}

Some suitable circuit configurations satisfying the above conditions can be found in Liu et al. [4], which describes a family of two-converter configurations for achieving two control functions. A minimal configuration is shown in Fig. 3. Note that the choice of the exact types of converters 1 and 2 remains a design issue.

To synthesize a desired impedance $Z(s)$, we have to ensure the correct magnitude and phase relationships between $v_{i}$ and $i_{i}$. Essentially we want $i_{i}$ to follow a reference template $i_{\text {ref }}$ which is related to $v_{i}$ as follows, in the complex frequency domain:

$$
I_{\text {ref }}(s)=V_{i}(s) G(s)
$$

where $G(s)$ is the transfer function from the input voltage to the reference template. Assume that the transfer function from $I_{\text {ref }}$ to $I_{i}$ is

$$
K(s)=\frac{I_{i}(s)}{I_{\mathrm{ref}}(s)} .
$$

\footnotetext{
${ }^{3}$ This is exactly what a PFC converter does.

${ }^{4} \mathrm{~A}$ single converter having unity power factor cannot provide perfect dc output regulation because $v_{i} i_{i} \neq v_{o} i_{o}$, where $v_{i} i_{i}$ is square of sine and $v_{o} i_{o}$ is constant.
} 


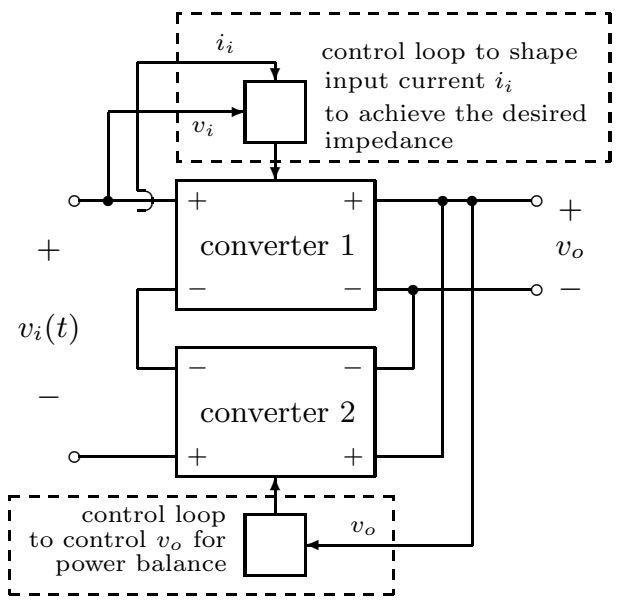

Figure 3: A minimal configuration, with at least one low-frequency storage within either converter 1 or 2 .

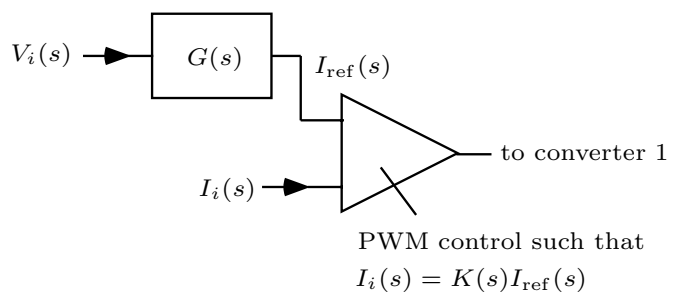

Figure 4: Current shaping control for achieving the required magnitude and phase relationships between input voltage and input current. $K(s)$ is nearly constant if all variables are slowly varying relative to the switching frequency.

Suppose $Z(s)$ is to be synthesized. Then, $G(s)$ becomes

$$
G(s)=\frac{1}{K(s) Z(s)} .
$$

Thus, in the circuit implementation, we have to realize this transfer function in order to synthesize the required impedance. A block diagram showing the control requirement is shown in Fig. 4.

Clearly, $K(s)$ in general depends on the converter response. However, if the bandwidth of the converter response is much higher than that of the applied voltage $v_{i}$, we may assume that $K(s)$ is nearly a constant, i.e., $i_{i}$ is proportional to $i_{\text {ref }}$. Thus, we can find the control transfer function $G(s)$ from (5).

\section{Example1: Inductive Impedance Synthesis}

The impedance of a series connection of an inductor $L$ and a resistor $R_{L}$ is

$$
Z_{L R}(s)=s L+R_{L}
$$

and $G(s)$ can be calculated according to (5) as

$$
G(s)=\frac{1}{K R_{L}\left(1+\frac{s L}{R_{L}}\right)} .
$$

The transfer function $G(s)$ is a single pole system and can be easily implemented [5].

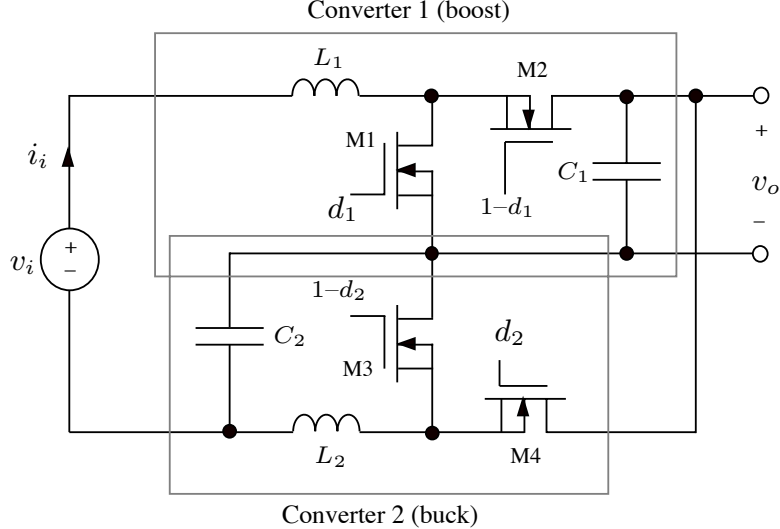

Figure 5: Experimental impedance synthesizer based on the configuration of Fig. 3, with converter 1 and converter 2 realized by a boost converter and a buck converter, respectively. $C_{2}$ serves as internal storage.

\section{Example 2: Capacitive Impedance Synthesis}

The impedance of a parallel connection of a capacitor $C$ and a resistor $R_{C}$ is

$$
Z_{C R}(s)=\frac{R_{C}}{1+s C R_{C}}
$$

and hence $G(s)$ can be calculated according to (5) as

$$
G(s)=\frac{1}{K\left(\frac{R_{C}}{1+s C R_{C}}\right)}=\frac{1}{K} \frac{1+s C R_{C}}{R_{C}} .
$$

The transfer function $G(s)$ is a one zero system and can also be readily implemented [5].

\section{EXPERIMENTAL RESULTS}

A particular choice of practical converter types for the implementation of the impedance synthesizer is shown in Fig. 5. Here, the input $v_{i}$ is a sinusoidal voltage source, and the output $v_{o}$ is connected to a current load. The switching frequency is $50 \mathrm{kHz}$. The two examples described in the previous section are evaluated.

The first impedance to be synthesized is $L=0.3 \mathrm{H}$ in series with $R_{L}=50 \Omega$. We have realized $G(s)$ using op-amps and discrete components. The transfer function $K$ was found to be $1.18 \mathrm{~A} / \mathrm{V}$. Figure 6 (a) shows the measured waveforms with $V_{i}(\mathrm{CH} 1)=80 \mathrm{~V}(\mathrm{rms})$ $50 \mathrm{~Hz}$ AC. CH4 shows the current $I_{i}$ with inductive characteristics of lagging phase. The measured phase lag of $I_{i}$ is $54.72^{\circ}$ and the amplitude is $0.8436 \mathrm{~A}(\mathrm{rms})$. The measured synthesized impedance is equivalent to $L=0.246 \mathrm{H}$ and $R_{L}=54.772 \Omega$ in series and is close to the designed values of $L=0.28 \mathrm{H}$ and $R_{L}=45.107 \Omega$. The error is expected to be caused by the phase difference shown in Fig. 5 (b) of $I_{\text {ref }}(\mathrm{CH} 3)$ and $I_{i}$, which has been ignored in the calculation of $G(s)$.

Next, we synthesize a parallel connection of $C=$ $22 \mu \mathrm{F}$ and $R_{C}=300 \Omega$. Figure 7 (a) shows the measured waveforms with $V_{i}(\mathrm{CH} 1)=80 \mathrm{~V}(\mathrm{rms}) 50 \mathrm{~Hz}$ AC. $\mathrm{CH} 4$ shows the current $I_{i}$ with capacitive characteristics 


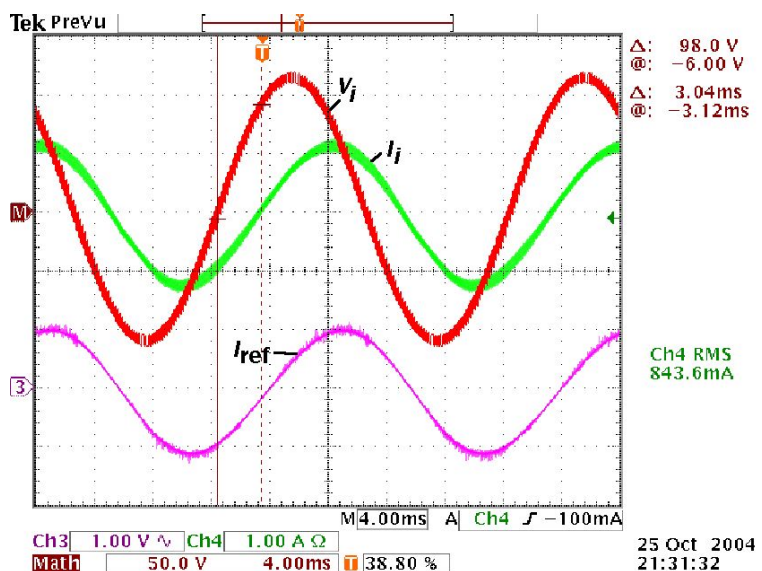

(a)

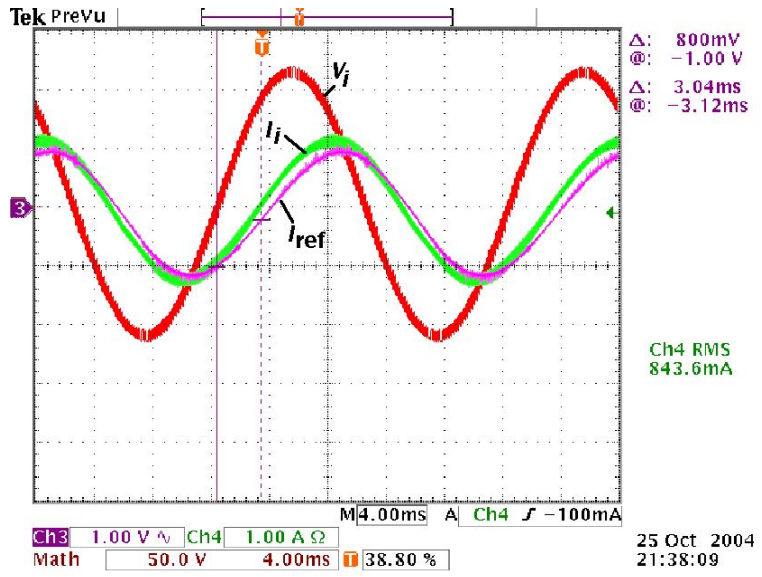

(b)

Figure 6: Measured input current and voltage from inductive impedance synthesizer showing (a) phase lead of voltage; (b) small phase difference between $I_{\text {ref }}$ and input current. Impedance being synthesized is $50 \Omega$ in series with $0.3 \mathrm{H}$.

of leading phase. The measured synthesized impedance is equivalent to $C=18.1 \mu \mathrm{F}$ and $R=242.6 \Omega$ in parallel connection and is close to the designed values of $C=21.24 \mu \mathrm{F}$ and $R=291.902 \Omega$. The error is also expected to be caused by the phase difference of $I_{\text {ref }}$ and $I_{i}$ shown in Fig. 7 (b).

\section{CONCLUSIONS}

A general impedance synthesis scheme using high frequency switching converters is proposed. With this method, we can imitate any impedance for control purpose, such as in power factor correction. It is also possible to synthesize impedances such as negative inductor and negative capacitor which are not generally available as circuit components.

\section{ACKNOWLEDGMENTS}

This work is supported by Hong Kong Research Grant Council under a CERG Grant (No. PolyU 5237/04E).

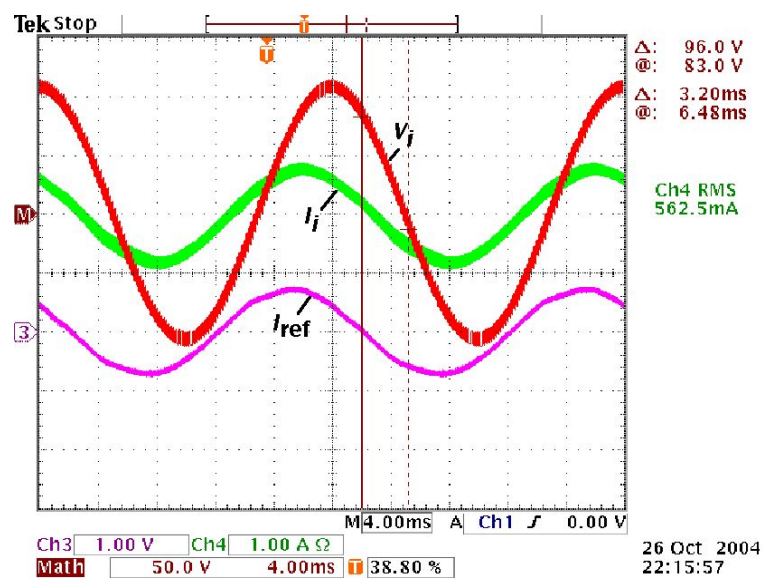

(a)

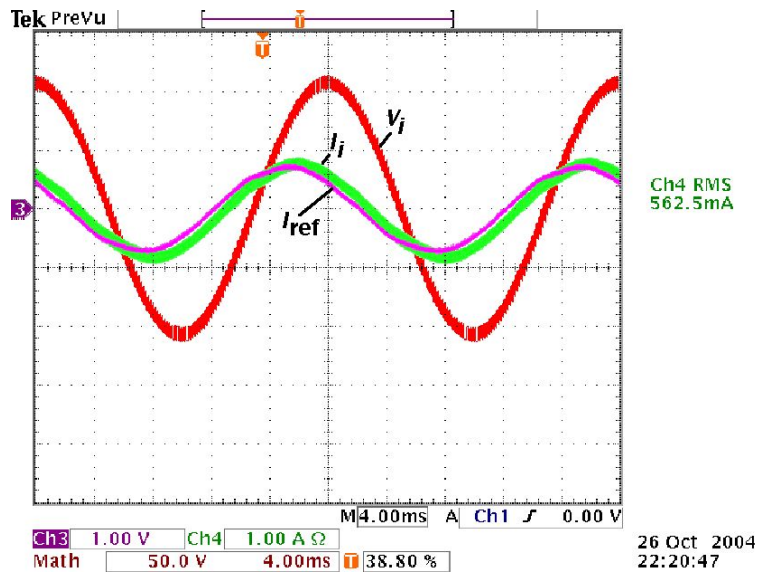

(b)

Figure 7: Measured input current and voltage from capacitive impedance synthesizer showing (a) phase lead of current; (b) small phase difference between $I_{\text {ref }}$ and input current. Impedance being synthesized is $300 \Omega$ in parallel with $22 \mu \mathrm{F}$.

\section{References}

[1] T. Nishi, "On the transformerless synthesis of reactance two-ports of degree four or less," IEEE Trans. Circ. Syst. I, vol. 15, no. 2, pp. 132-138, 1988.

[2] C.K. Tse, "Circuit theory of power factor correction in switching converters," Int. J. Circuit Theory Appl., vol. 31, no. 2, pp. 157-198, March 2003.

[3] N.K. Poon, C.K. Tse and J. Liu, "Very fast transient voltage regulators based on load correction," IEEE Power Electron. Specialists Conf. Record, pp. 66-71, June 1999.

[4] C.P. Liu, C.K. Tse, N.K. Poon, M.H. Pong and Y.M. Lai, "Synthesis of input-rectifierless ac/dc converters," IEEE Trans. Power Electron., vol. 19, no. 1, pp. 176-182, January 2004.

[5] R. Schaumann, M.S. Ghausi and K.R. Laker, Design of Analog Filters: Passive, Active $R C$, and Switched Capacitor, Englewood Cliffs: Prentice Hall, 1990. 